\title{
Tridiagonal GPU Solver with Scaled Partial Pivoting at Maximum Bandwidth
}

\author{
Christoph Klein \\ Robert Strzodka \\ christoph.klein@ziti.uni-heidelberg.de \\ robert.strzodka@ziti.uni-heidelberg.de \\ Institute of Computer Engineering (ZITI) \\ Heidelberg, Germany
}

\begin{abstract}
Partial pivoting is the method of choice to ensure stability in matrix factorizations performed on CPUs. For sparse matrices, this has not been implemented on GPUs so far because of problems with datadependent execution flow. This work incorporates scaled partial pivoting into a tridiagonal GPU solver in such a fashion that despite the data-dependent decisions no SIMD divergence occurs. The cost of the computation is completely hidden behind the data movement which itself runs at maximum bandwidth. Therefore, the cost of the tridiagonal GPU solver is no more than the minimally required data movement. For large single precision systems with $2^{25}$ unknowns, speedups of 5 are reported in comparison to the numerically stable tridiagonal solver (gtsv2) of cuSPARSE. The proposed tridiagonal solver is also evaluated as a preconditioner for Krylov solvers of large sparse linear equation systems. As expected it performs best for problems with strong anisotropies.
\end{abstract}

\section{CCS CONCEPTS}

- Mathematics of computing $\rightarrow$ Solvers; Mathematical software performance.

\section{KEYWORDS}

tridiagonal solver, GPUs, CUDA, iterative solver, preconditioner

\section{ACM Reference Format:}

Christoph Klein and Robert Strzodka. 2021. Tridiagonal GPU Solver with Scaled Partial Pivoting at Maximum Bandwidth. In 50th International Conference on Parallel Processing (ICPP '21), August 9-12, 2021, Lemont, IL, USA. ACM, New York, NY, USA, 10 pages. https://doi.org/10.1145/3472456.3472484

\section{INTRODUCTION}

Solving a tridiagonal system represents a common problem in many scientific and engineering problems, e.g. fluid dynamics [20, 24], cubic spline interpolation [6], electrodynamics [21], semicoarsening for multigrid solvers [28], preconditioning for multigrid solvers [17], or computer graphics [23]. A fast and stable tridiagonal solver represents an important building block for these applications.

Permission to make digital or hard copies of part or all of this work for personal or classroom use is granted without fee provided that copies are not made or distributed for profit or commercial advantage and that copies bear this notice and the full citation on the first page. Copyrights for third-party components of this work must be honored

For all other uses, contact the owner/author(s).

ICPP '21, August 9-12, 2021, Lemont, IL, USA

(C) 2021 Copyright held by the owner/author(s).

ACM ISBN 978-1-4503-9068-2/21/08.

https://doi.org/10.1145/3472456.3472484
The classical sequential algorithms for tridiagonal systems, the Thomas Algorithm [31], cannot be executed in parallel. The most well known parallel algorithms to solve tridiagonal systems are the Cyclic Reduction [21] (CR) the Parallel Cyclic Reduction [22] (PCR), the Partition Method [33], and the Divide and Conquer [34] approach, which were initially implemented on classical vector machines and more recently implemented on GPUs. Although many tridiagonal solvers for systems of small size, which fit into on-chip memory (CUDA shared memory), for GPUs exist [11, 16, 26, 30], only a few numerically stable solvers for large systems exist $[5,12$, 32], but none of them uses (scaled) partial pivoting.

This paper presents a solver with scaled partial pivoting for large tridiagonal systems, which is based on a partition method. The new contributions of the solver are

- data-dependent scaled partial pivoting without SIMD divergence,

- minimal pivot encoding with one bit per row produced and consumed on-chip,

- minimal data movement with on-the-fly data transposition at maximum bandwidth,

- very little additional memory is allocated by the solver, which allows maximally large tridiagonal systems on the GPU.

The remaining limitation is a general problem of static partition methods that we do not explicitely control the condition of the coarse system. This may result in ill-conditioned coarse systems, which negatively affect the overall accuracy of the solution. In practice, a sensitivity to the chosen partitioning is rather seldom, but an explicit control would still be desirable.

Besides the performance aspects of the tridiagonal solver alone, the paper also analyzes the performance of Krylov iterative solvers with this new tridiagonal solver as a preconditioner, which is a common choice for anisotropic problems $[4,18]$.

The paper is structured as follows: Section 2 summarises related work, Section 3 gives a detailed description of the proposed tridiagonal solver, Section 4 evaluates the tridiagonal solver as a preconditioner.

\section{RELATED WORK}

GPU implementations for CR and PCR were done by many authors $[1,8,17,23,25]$ and used as a smoother for a multigrid solver by Göddeke and Strzodka [17]. For the first time, a numerically stable tridiagonal solver for the GPU was implemented by Chang et al. [5, 7], which uses diagonal pivoting by Erway et al. [15]. Chang's implementation was improved regarding the numeric stability by Venetis et al. [32], which point out the problematic behavior of 
diagonal pivoting in combination with singular submatrices and therefore proposed g-Spike. Wang et al. [35] implemented SPIKE ${ }^{2}$ to solve large-scale tridiagonal systems on CPU-GPU hybrid systems. This improves the implementation of the tiled-PCR-p-Thomas algorithm by Kim et al. [25]. The T-SCG solver by Li et al. [27] is a combination of $\mathrm{SPIKE}^{2}$ and the diagonal pivoting technique. Recently Diéguez et al. [10-14] proposed two tridiagonal solvers: the Tree Partitioning Reduction (TPR) and a Wang and Mou implementation with their Butterfly Processing Library for GPUs (WM BPLG).

\section{TRIDIAGONAL SOLVER}

In this paper, the Recursive Partitioned Tridiagonal Schur Complement Algorithm with scaled partial pivoting (RPTS) is proposed, which solves

$$
A x=d,
$$

where $A$ is a tridiagonal matrix. Viewed as a graph a tridiagonal system is a long chain of connected nodes as shown in Figure 1 on the left. We partition this chain into regular partitions of size $M$ with 2 interface nodes and $M-2$ inner nodes in each partition. Let $I$ be the index set of all interface nodes and $P$ be the index set of all inner partition nodes. All coefficients in matrix $A$ can then be classified as connections within or between these index sets: the $(M-2) \times(M-2)$-block diagonal $A_{P P}$, the $2 \times 2$-block diagonal $A_{I I}$, the tall $A_{P I}$, and the wide $A_{I P}$. The corresponding Schur-complement factorization reads:

$$
\begin{gathered}
A=\left(\begin{array}{cc}
A_{P P} & A_{P I} \\
A_{I P} & A_{I I}
\end{array}\right)=\left(\begin{array}{cc}
I & 0 \\
A_{I P} A_{P P}^{-1} & I
\end{array}\right)\left(\begin{array}{cc}
A_{P P} & A_{P I} \\
0 & S
\end{array}\right) \\
S:=A_{I I}-A_{I P} A_{P P}^{-1} A_{P I}
\end{gathered}
$$

The above factorization with the Schur-complement $S$ allows the recursive solution of

$$
A x=\left(\begin{array}{ll}
A_{P P} & A_{P I} \\
A_{I P} & A_{I I}
\end{array}\right)\left(\begin{array}{l}
x_{P} \\
x_{I}
\end{array}\right)=\left(\begin{array}{l}
d_{P} \\
d_{I}
\end{array}\right)=d
$$

in three steps

Reduce: Compute the coarse system $S$ and the new right-hand side $d_{I}^{\prime}:=d_{I}-A_{I P} A_{P P}^{-1} d_{P}$.

Solve coarse system: Solve $S x_{I}=d_{I}^{\prime}$ by recursively applying the partitioning and factorization to $S$.

Substitute: Back-substitute $x_{I}$ to obtain $d_{P}^{\prime}:=d_{P}-A_{P I} x_{I}$ and solve for the inner nodes $x_{P}=A_{P P}^{-1} d_{P}^{\prime}$.

For a general $A$, the Schur-complement $S$ has a different sparsity pattern than $A$, in fact $S$ is usually dense. However, in this special case $S$ is also tridiagonal, so we can recursively partition and factorize $S$ in the same fashion as $A$ until we arrive at a very small tridiagonal system which is solved directly. During the reduction step the inner of each partition is diagonalized, which produces fill-ins in the left-most and right-most columns, as seen after step I in Figure 1. For small tridiagonal systems without pivoting this diagonalization pattern was also used by Giles et al. [16] and further discussed by László et al. [26]. Our diagonalization of the reduction phase has an increased amount of parallelism because the upwards and downwards oriented elimination is calculated in parallel.
After one reduction step, a smaller tridiagonal system is obtained with the size of $N^{\prime}=2 N / M$, which is marked in yellow in Figure 1. In the following, the smaller system is referred to as the coarse system and the input system as the fine system. The reduction kernel of RPTS is applied recursively, until the size of the system is sufficiently small, to be solved directly (step III in Figure 1). The size of the coarse system is only a small fraction of the fine system, e.g. for $M=37$ the size of coarse system is just $5 \%$ of the fine system. Thus from the computational point of view, increasing $M$ further hardly yields any benefits. If $N$ is not a multiple of $M$, the size of the last partition is $(N \bmod M)$.

During the substitution phase, the solution of the coarse system is already known, and the corresponding values can be substituted, which is shown in Figure 1 after step IV. The green values are known and can be substituted. In the substitution phase, the diagonalization is recalculated without fill-ins because the substitutions from the coarse system make each partition of the fine system independent of each other. The recalculation obviously incurs additional arithmetic operations, but we minimize the data movement because neither the diagonalized system nor the permutation (pivot locations) must be written to memory after the reduction step. Since RPTS is still a bandwidth limited algorithm, trading additional computation for minimal data movement is beneficial. In particular, on GPUs with so many compute units, the additional calculations can be hidden behind the memory movement operations.

Algorithm 1 sketches the calculation of the reduction phase for one partition (see Figure 1); in the actual implementation many partitions are treated in parallel in this way. The indexing starts with zero and refers to the starting row of the current partition. $a, b$, $c, d$ are vectors of length $M$, which represent the lower, middle, and upper band; and the right-hand side vector. $s_{c}$ and $s_{p}$ are temporary register arrays and denote the current and previous set of values, respectively (s[0] is the spike, $s[4]$ is the right-hand side). $r_{c}, r_{p}, m_{c}$, and $m_{p}$ are scalar values. The function reverse_view reverses the view on the ranges $a, b, c$, or $d$ (e.g. reverse_view $(a)[0]=a[M-1]$ ). We use ';' as a delimiter between different instructions on the same line. $\epsilon$ is a threshold parameter, and $\tilde{\epsilon}$ is the smallest representable value in the current data format. The function apply_threshold maps coefficients smaller than $\epsilon$ to zero. This option allows the user to increase numeric stability in the case of noisy input coefficients. Setting $\epsilon=0$ switches off this behavior. In line 27 and 28 the function eliminate_band is called in parallel for the upwards and downwards oriented elimination, which is possible because nothing needs to be saved in memory during the elimination.

Algorithm 2 shows the substitution phase, which cannot execute the downwards and upwards oriented elimination in parallel. During the downwards oriented elimination, the row indices of the pivots must be saved on-chip so that the upwards oriented substitution finds the coefficients with respect to the correct pivot locations. As each interface has two nodes, the solution of $x[M-2]$ and $x[1]$ can be obtained in two different ways, which is implemented in lines 24-28 and lines 34-38 of Algorithm 2, where the selection follows the partial pivoting criteria. 

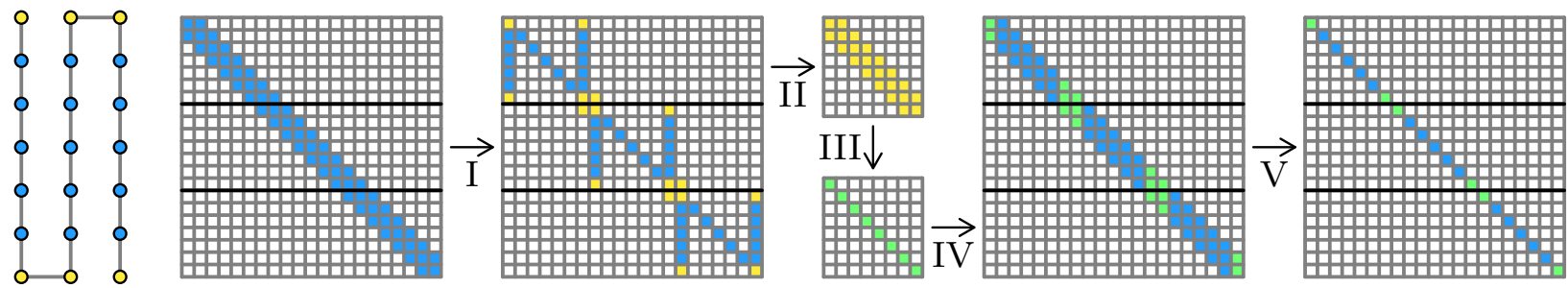

Figure 1: Matrix graph representation and matrix patterns during different phases of RPTS assuming no row permutations took place. The partition size $M$ is equal to 7 and the system size is $N=21$.

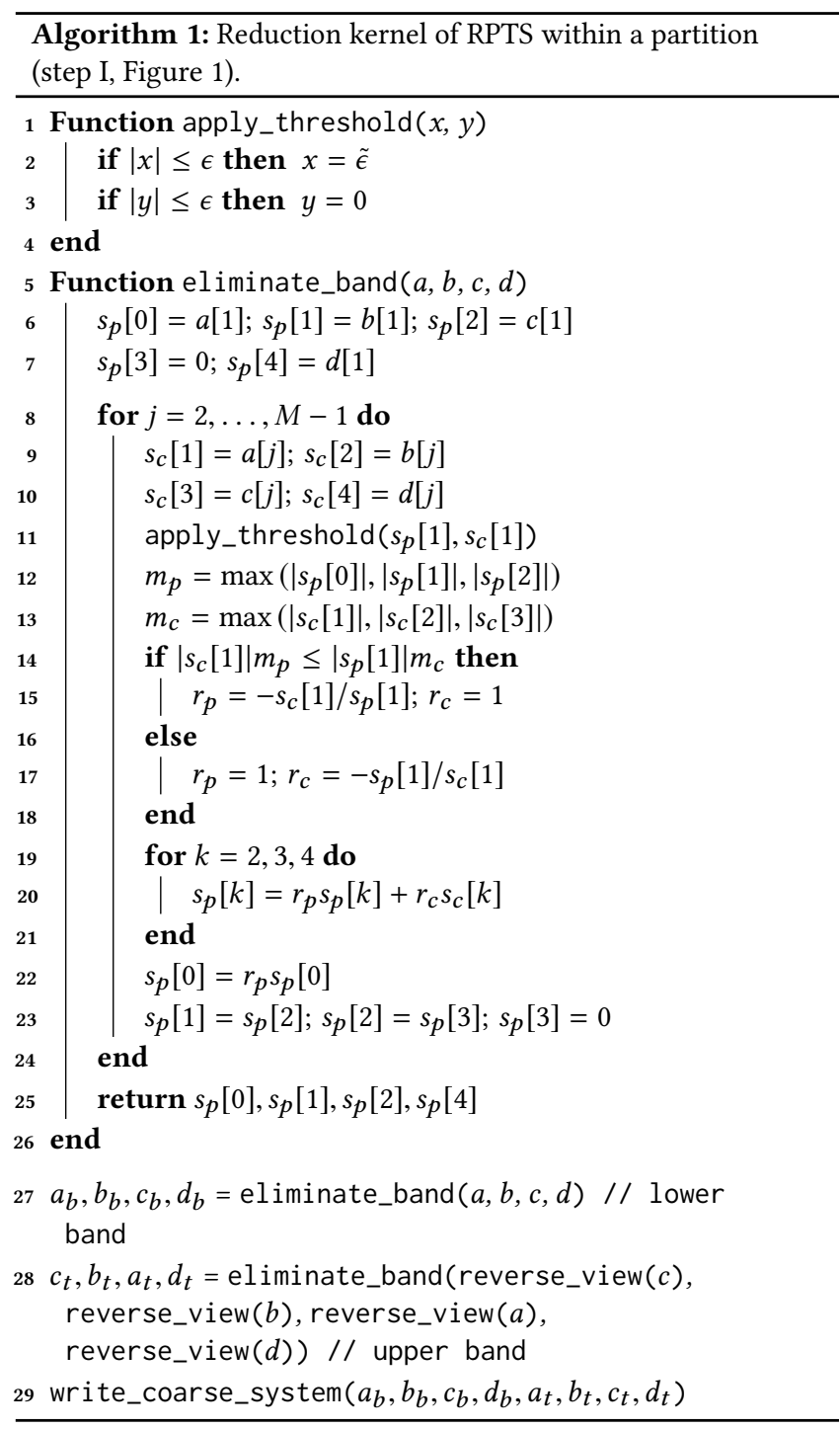

The pivoting of the Algorithms can be changed by chosing $m_{p}$ and $m_{c}$ accordingly

no pivoting $m_{p}=m_{c}=0$

partial pivoting $m_{p}=m_{c}=1$

scaled partial pivoting shown in Algorithm 1 and 2

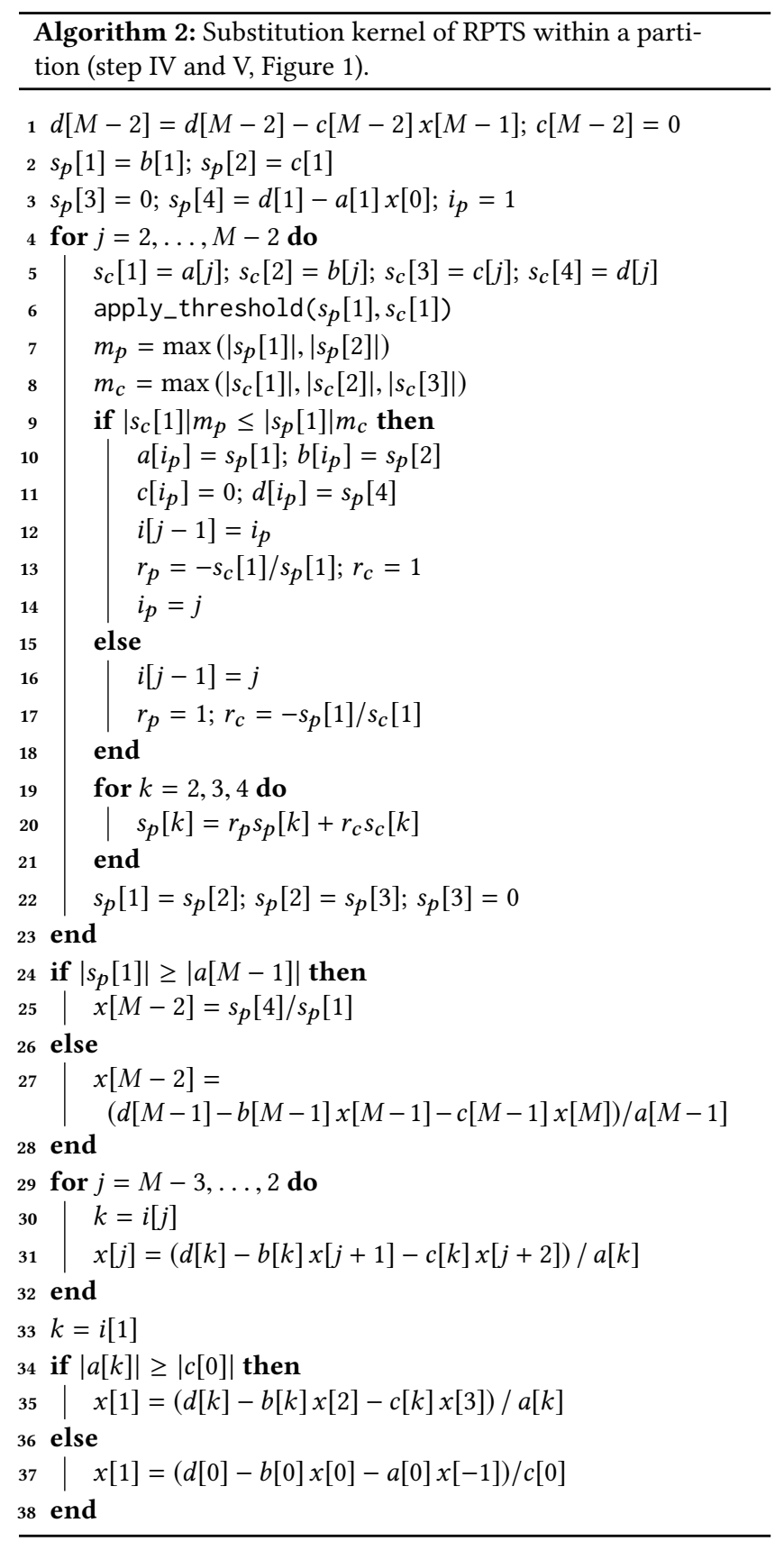




\subsection{Implementation}

The tridiagonal solver has been implemented in CUDA with such algorithmic optimizations that all data is moved at maximum bandwidth and for sufficiently large systems the entire computation is hidden behind memory operations, i.e. we only see the cost of reading and writing data from global memory to the GPU.

3.1.1 Data Layout. RPTS expects the tridiagonal input matrix $A$ in the band format, analogously to NVIDIA's implementation of a tridiagonal solver in cuSPARSE. Each band $(a, b, c)$ is saved separately in a buffer of length $N$. The unused $a[0]$ and $c[N-1]$ are set to zero. Apart from the input data RPTS allocates very little extra memory for the hierarchy of coarse tridiagonal systems and vectors, e.g. for $N=2^{25}, M=41$ the overall additional memory is only $5.13 \%$ of the input data.

3.1.2 Shared Memory Layout. To ensure full memory bandwidth the threads must load the bands and right-hand side of their partitions in a coalesced way into shared memory. Figure 2a shows an example for one thread block of 6 threads, which load their data into shared memory. Subsequently, Figure $2 \mathrm{~b}$ demonstrates that during the elimination each thread processes the values of its partition (with size $M=7$ ) sequentially. Thread 0 reads element 0 while thread 1 reads element 7 . All threads participate in reading and writing data, but not necessarily all threads of a block participate in the elimination process due to the fact that the number of partitions per block $L$ is another tuning parameter. Generally, $L=32$ is already sufficient because then one full CUDA warp calculates the elimination. The example in Figure 2 shows that only 5 threads process a partition, although 6 threads load the data into shared memory. In general $N$ is not divisible by $M$ and the last thread in the last block, which participates in the elimination process, processes less than $M$ equations.

The overall shared memory consumption per block is $4 M \cdot L$ elements, i.e. the three bands $a, b, c$ plus right-hand side $d$. During the substitution phase it is necessary to save the solution $x$ in shared memory, but only $2 L$ additional shared memory elements must be occupied because $x$ may reuse the buffer for the right-hand side.

3.1.3 Storage format for row interchanges during pivoting. The array, which saves pivot locations, denoted by $i[j]$ in Algorithm 2 requires $M$ entries. Thus if the array is saved trivially as indices, additional memory of $M \cdot L$ integers would be required for each CUDA thread block. This could be saved in either shared memory or registers. If $i[j]$ were saved in shared memory, the overall shared memory consumption per CUDA thread block would increase unneccassarily limiting the maximum possible value for $M$. Saving $i[j]$ in registers, on the other hand, would decrease the occupancy and thus the latency hiding ability of the GPU. Therefore, we develop a minimal storage format for the pivot locations.

When a thread calculates the downwards oriented elimination, there are only two options, the current or the previous row is scaled and added to create a zero in the column $j$. Hence it is sufficient to use only one bit to distinguish between these two options, and $M$ bits are required to save the pivoting locations for one partition. During the upwards oriented substitution, the actual index $i[j]$ is efficiently reconstructed from the bit pattern with bitwise operations. In the current implementation, a long long int with 64 bits saves the bit pattern. This limits $M$ to 64 , however, larger values of $M$ are not required, because the size of the coarse system is already negligible in comparison to the fine system.

3.1.4 Complete avoidance of SIMD divergence. At first look, Algorithm 1 and 2 appear to have an overly complicated access to the bands and values of $a, b, c, d$ through $s_{p}$ [0], $s_{p}$ [1], $s_{p}[2], s_{p}$ [3], $s_{c}[0], s_{c}[1], s_{c}[2], s_{c}[3]$. This is the result of a carefully crafted algorithmic formulation of the elimination in which all data-dependent conditionals assume the form of a value selection

$$
\text { result = condition ? value } 1 \text { : value } 0
$$

and only a little predication is required in the computation of value 1 and value 0 . Note, the writing of the coefficients in lines 10 and 11 in Algorithm 2 can be placed in front of the if-statement at the cost of writing them redundantly in every iteration. Therefore, every if-statement is replaced with the aforementioned value selection in the implementation. Consequently, the profiler reports zero SIMD divergence despite all threads making data-dependent pivoting decisions in parallel.

3.1.5 Avoidance of shared memory bank conflicts. The reduction kernel is completely free of shared memory bank conflicts because all pivoting decisions are processed in registers and the elimination of the lower and upper band is done by two different CUDA warps independently. If $M$ is even, the shared memory arrays are padded by 1 ensuring zero bank conflicts in the parallel access. In the substitution kernel, bank conflicts cannot be avoided completely because the access to shared memory depends on the pivot locations.

\subsection{Results}

All results presented in this paper have been computed on a machine with CentOS 7, CUDA Toolkit 11.0.167, CUDA driver 450.57, host compiler GCC 8.3.0, and a GeForce RTX 2080 Ti or a GeForce GTX 1070 GPU.

RPTS is compared against four other numerically stable tridiagonal solvers: First, cuSPARSE (gtsv2), which according to Venetis et al. [32] was a SPIKE implementation for GPUs by Chang et al. [5, 7] with diagonal pivoting [15]. Currently, the cuSPARSE documentation is not stating the specific algorithm, which is used by gtsv2, but the kernel names shown by the CUDA profiler, indicate that the aforementioned algorithm is still in use. Second, g-spike by Venetis et al. [32], which is an improvement regarding the numeric stability of the SPIKE implementation by Chang et al. [7]. Third, LAPACK's tridiagonal solver (gtsv). And fourth, the sparse LU decomposition implemented in Eigen3 [19].

There are four parameters, which control the numerical results of RPTS: First, the partition size $M$, second, the upper limit for the system size $\tilde{N}$, which is solved directly (see Figure 1 step III), third, the threshold parameter $\epsilon$, and fourth, the solver, which is used to solve the coarsest system directly. In the following results $M=32$, $\tilde{N}=32, \epsilon=0$ and a single CUDA thread with an adjusted version of Algorithm 2 is used to solve the coarsest system.

Following the literature [7, 12, 27, 32], we use the matrices listed in Table 1 as test cases for the numerical evaluation. For the size of the matrices, $N=512$ is chosen, and all calculations are done in double-precision. Table 2 shows the forward relative error, which is equal to $\left|x-x_{t}\right|_{2} /\left|x_{t}\right|_{2}$, where $x_{t}, x$ denote the true and calculated 


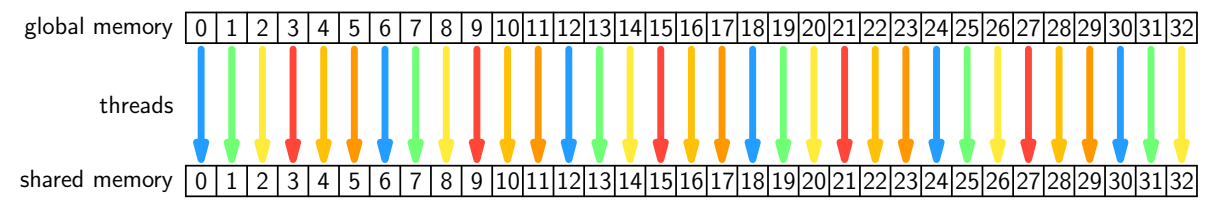

(a) Loading into shared memory

shared memory \begin{tabular}{|l|l|l|l|l|l|l|l|l|l|l|l|l|l|l|l|l|l|l|l|l|l|l|l|l|l|l|l|l|l|l|l|l|}
\hline 0 & 1 & 2 & 3 & 4 & 5 & 6 & 7 & 8 & 9 & 10 & 11 & 12 & 13 & 14 & 15 & 16 & 17 & 18 & 19 & 20 & 21 & 22 & 23 & 24 & 25 & 26 & 27 & 28 & 29 & 30 & 31 & 32 \\
\hline
\end{tabular}

(b) Processing in shared memory

Figure 2: Data transposition in shared memory of RPTS. A band (e.g. $a_{i}$ ) is loaded coalesced into shared memory and subsequently processed sequentially by a thread; different threads appear in different colors.

\begin{tabular}{|c|c|c|}
\hline ID & condition number & Description \\
\hline 1 & $1.58 \mathrm{e}+03$ & $\operatorname{tridiag}(a, b, c)$ with $a, b, c$ sampled from $U(-1,1)$ \\
\hline 2 & $1.00 \mathrm{e}+00$ & $\mathrm{~b}=1 \mathrm{e}+8 *$ ones $(\mathrm{N}, 1) ; \mathrm{a}, \mathrm{c}$ sampled from $U(-1,1)$ \\
\hline 3 & $3.52 \mathrm{e}+02$ & gallery('lesp', N) \\
\hline 4 & $2.93 e+03$ & same as $\# 1$, but $a(N / 2+1, N / 2)=1 e-50 * a(N / 2+1, N / 2)$ \\
\hline 5 & $1.59 \mathrm{e}+03$ & same as \#1, but each element of a, c has $50 \%$ chance to be zero \\
\hline 6 & $1.04 \mathrm{e}+00$ & $\mathrm{~b}=64 *$ ones $(N, 1) ; \mathrm{a}$, c sampled from $U(-1,1)$ \\
\hline 7 & $9.00 \mathrm{e}+00$ & inv(gallery ('kms', N, 0.5)) Toeplitz, inverse of Kac-Murdock-Szegö \\
\hline 8 & $1.02 \mathrm{e}+15$ & gallery ('randsvd', $N, 1 \mathrm{e} 15,2,1,1$ ) \\
\hline 9 & $8.74 \mathrm{e}+14$ & gallery ('randsvd', $\mathrm{N}, 1 \mathrm{e} 15,3,1,1$ ) \\
\hline 10 & $1.11 \mathrm{e}+15$ & gallery ('randsvd', $\mathrm{N}, 1 \mathrm{e} 15,1,1,1$ ) \\
\hline 11 & $9.57 \mathrm{e}+14$ & gallery('randsvd', $\mathrm{N}, 1 \mathrm{e} 15,4,1,1)$ \\
\hline 12 & $3.07 \mathrm{e}+23$ & same as $\# 1$, but $a=a * 1 e-50$ \\
\hline 13 & $1.40 \mathrm{e}+17$ & gallery('dorr', $N, 1 e-4)$ \\
\hline 14 & $8.17 \mathrm{e}+14$ & tridiag (a, $1 \mathrm{e}-8 *$ ones $(\mathrm{N}, 1), \mathrm{c})$ with a, c sampled from $U(-1,1)$ \\
\hline 15 & $2.15 \mathrm{e}+20$ & $\operatorname{tridiag}(a, \operatorname{zeros}(N, 1)$, c) with a, c sampled from $U(-1,1)$ \\
\hline 16 & $3.27 \mathrm{e}+02$ & tridiag (ones $(\mathrm{N}-1,1), 1 \mathrm{e}-8 *$ ones $(\mathrm{N}, 1)$, ones $(\mathrm{N}-1,1)$ ) \\
\hline 17 & $1.00 \mathrm{e}+00$ & tridiag (ones $(\mathrm{N}-1,1), 1 \mathrm{e} 8 *$ ones $(\mathrm{N}, 1)$, ones $(\mathrm{N}-1,1)$ ) \\
\hline 18 & $3.00 \mathrm{e}+00$ & $\operatorname{tridiag}(-\operatorname{ones}(\mathrm{N}-1,1), 4 * \operatorname{ones}(\mathrm{N}, 1),-\operatorname{ones}(\mathrm{N}-1,1))$ \\
\hline 19 & $1.12 \mathrm{e}+00$ & $\operatorname{tridiag}(-$ ones $(\mathrm{N}-1,1), 4 *$ ones $(\mathrm{N}, 1)$, ones $(\mathrm{N}-1,1))$ \\
\hline 20 & $2.30 \mathrm{e}+00$ & $\operatorname{tridiag}(-$ ones $(\mathrm{N}-1,1), 4 *$ ones $(\mathrm{N}, 1), \mathrm{c})$, c sampled from $U(-1,1)$ \\
\hline
\end{tabular}

Table 1: Tridiagonal matrix collection for numerical-stability analysis (taken from [32]). MATLAB functions are used. Function $\operatorname{tridiag}(a, b, c)$ returns a tridiagonal matrix with main diagonal $b$ and sub and superdiagonals a and $c . U(-1,1)$ is the uniform distribution between one and minus one. All matrices are of the same size $N$. The condition number was calculated with the JacobiSVD function of the Eigen3 library for matrices of size $N=512$.

solution, respectively. $x_{t}$ is initialized with a normal distribution of floating-point numbers with a mean value of 3 and standard deviation of 1 .

The reduction kernel of RPTS reads $4 N$ elements, which are the three bands and the right-hand side, and writes $8 N / M$ elements of the coarse system. The substitution kernel reads $4 N+2 N / M$ elements, because only the solution of the coarse system is required, and writes the solution vector of length $N$. The throughput, which is shown in Figure 3, is measured with the nvprof for the GTX 1070 and with NVIDIA Nsight Compute for the RTX 2080 Ti. For comparison, the performance of a simple copy kernel, which just copies a certain amount of data is shown. Although the throughput of the copy kernel does not reach the theoretical memory bandwidth, the copy kernel performance usually displays the hardware performance limit for memory-bound algorithms. Whereas the copy kernel reads and writes $N$ elements, this is different for the kernels of RPTS, which read more data than they write. Therefore higher throughputs than for the copy kernel are possible. In addition, the performance of the pure data movement without any calculation in the kernels of RPTS is shown for comparison. Processing the finest system heavily dominates the overall runtime. All coarse stages combined increase the overall runtime by only $8.5 \%$ for $N=2^{25}$.

By comparing the kernels with and without calculation we see that for sufficiently large tridiagonal systems the computation is completely hidden behind data movement. Only for smaller problem sizes, the kernels of RPTS are slower than the data movement alone. The detailed throughput of RPTS was measured manually with CUDA events by using the mean kernel time of 700 kernel executions. All performance measurements use single-precision as the GTX/RTX graphics cards have only few double-precision units. 

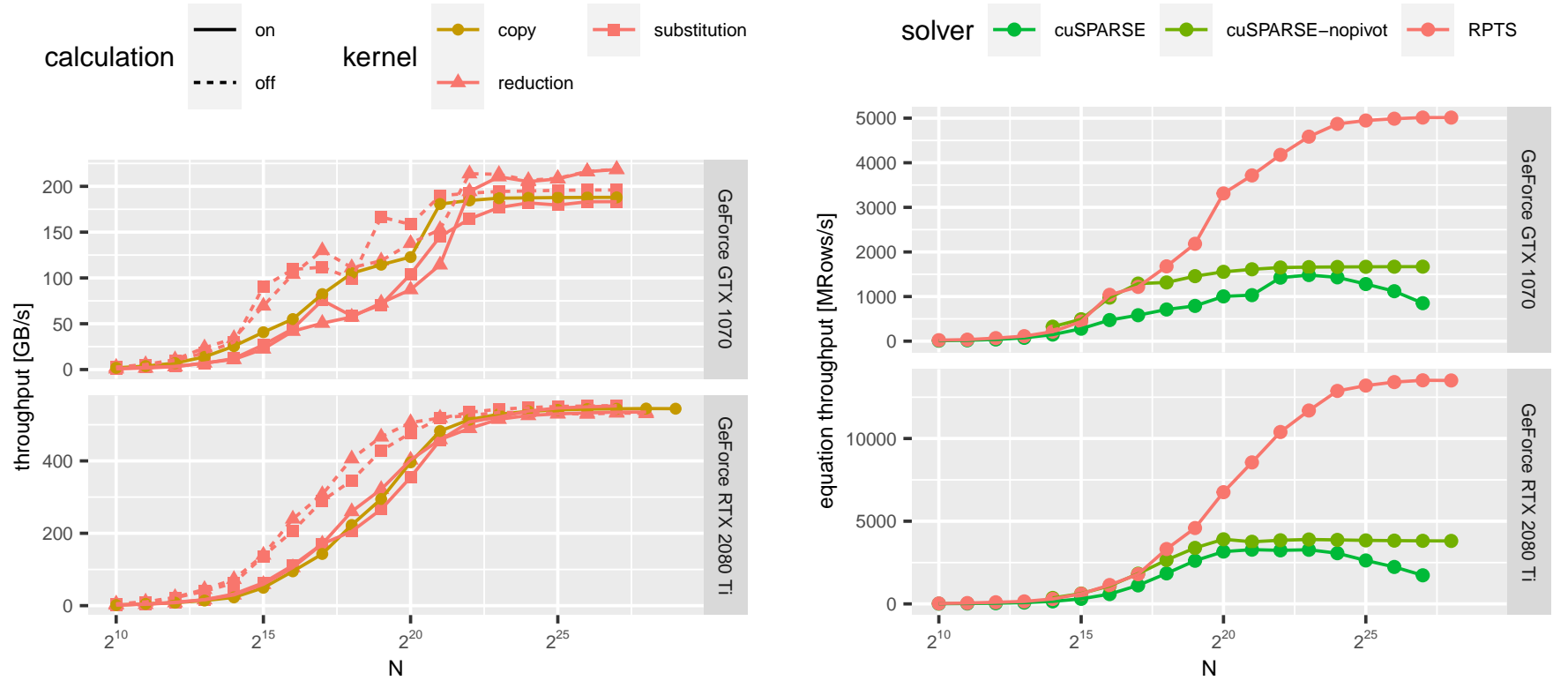

Figure 3: Single-precision performance of tridiagonal solvers for matrix 1 (see Table 1) with size N. Left: RPTS global memory throughput of the finest stage. Right: performance comparison to the tridiagonal solver from cuSPARSE. Missing data points indicate that the required memory exceeds the available GPU memory.

\begin{tabular}{crrrrr}
\hline matrix ID & Eigen3 & RPTS & cuSPARSE & g-spike & LAPACK \\
\hline 1 & $5.72 \mathrm{e}-15$ & $5.24 \mathrm{e}-15$ & $5.05 \mathrm{e}-15$ & $7.53 \mathrm{e}-15$ & $5.78 \mathrm{e}-15$ \\
2 & $8.39 \mathrm{e}-17$ & $8.32 \mathrm{e}-17$ & $1.18 \mathrm{e}-16$ & $1.30 \mathrm{e}-16$ & $8.39 \mathrm{e}-17$ \\
3 & $1.28 \mathrm{e}-16$ & $1.32 \mathrm{e}-16$ & $1.44 \mathrm{e}-16$ & $1.65 \mathrm{e}-16$ & $1.29 \mathrm{e}-16$ \\
4 & $5.62 \mathrm{e}-15$ & $5.25 \mathrm{e}-15$ & $6.17 \mathrm{e}-15$ & $1.55 \mathrm{e}-14$ & $6.12 \mathrm{e}-15$ \\
5 & $1.19 \mathrm{e}-15$ & $9.03 \mathrm{e}-16$ & $1.94 \mathrm{e}-15$ & $1.13 \mathrm{e}-15$ & $8.85 \mathrm{e}-16$ \\
6 & $9.33 \mathrm{e}-17$ & $9.57 \mathrm{e}-17$ & $1.32 \mathrm{e}-16$ & $1.50 \mathrm{e}-16$ & $9.33 \mathrm{e}-17$ \\
7 & $2.33 \mathrm{e}-16$ & $2.76 \mathrm{e}-16$ & $2.53 \mathrm{e}-16$ & $2.74 \mathrm{e}-16$ & $2.34 \mathrm{e}-16$ \\
8 & $1.18 \mathrm{e}-04$ & $4.53 \mathrm{e}-04$ & $1.29 \mathrm{e}-05$ & $5.52 \mathrm{e}-05$ & $1.26 \mathrm{e}-04$ \\
9 & $4.01 \mathrm{e}-05$ & $5.07 \mathrm{e}-05$ & $2.77 \mathrm{e}-05$ & $1.73 \mathrm{e}-05$ & $5.73 \mathrm{e}-05$ \\
10 & $4.66 \mathrm{e}-05$ & $1.25 \mathrm{e}-05$ & $1.85 \mathrm{e}-05$ & $4.88 \mathrm{e}-06$ & $5.19 \mathrm{e}-05$ \\
11 & $5.35 \mathrm{e}-05$ & $2.87 \mathrm{e}-04$ & $1.46 \mathrm{e}-03$ & $2.89 \mathrm{e}-03$ & $3.57 \mathrm{e}-04$ \\
12 & $9.45 \mathrm{e}+03$ & $1.35 \mathrm{e}+05$ & $7.63 \mathrm{e}+05$ & $2.51 \mathrm{e}+05$ & $9.45 \mathrm{e}+03$ \\
13 & $1.08 \mathrm{e}+00$ & $2.45 \mathrm{e}+00$ & $1.33 \mathrm{e}+00$ & $1.21 \mathrm{e}+00$ & $4.37 \mathrm{e}-01$ \\
14 & $1.08 \mathrm{e}-03$ & $1.76 \mathrm{e}-03$ & $2.89 \mathrm{e}-03$ & $9.05 \mathrm{e}-02$ & $1.28 \mathrm{e}-03$ \\
15 & $5.21 \mathrm{e}+02$ & $5.01 \mathrm{e}+02$ & $9.24 \mathrm{e}+02$ & $4.45 \mathrm{e}+02$ & $5.21 \mathrm{e}+02$ \\
16 & $8.67 \mathrm{e}-16$ & $1.37 \mathrm{e}-15$ & $3.49 \mathrm{e}-15$ & $3.89 \mathrm{e}-15$ & $7.75 \mathrm{e}-16$ \\
17 & $1.14 \mathrm{e}-16$ & $1.16 \mathrm{e}-16$ & $1.60 \mathrm{e}-16$ & $1.53 \mathrm{e}-16$ & $1.14 \mathrm{e}-16$ \\
18 & $8.94 \mathrm{e}-17$ & $1.04 \mathrm{e}-16$ & $1.36 \mathrm{e}-16$ & $1.42 \mathrm{e}-16$ & $8.94 \mathrm{e}-17$ \\
19 & $1.10 \mathrm{e}-16$ & $1.11 \mathrm{e}-16$ & $1.51 \mathrm{e}-16$ & $1.57 \mathrm{e}-16$ & $1.10 \mathrm{e}-16$ \\
20 & $1.18 \mathrm{e}-16$ & $1.11 \mathrm{e}-16$ & $1.46 \mathrm{e}-16$ & $1.51 \mathrm{e}-16$ & $1.17 \mathrm{e}-16$ \\
\hline
\end{tabular}

Table 2: Forward relative error of double-precision results for numerical-stability analysis of tridiagonal solvers.

Figure 3 shows the equation throughput with a partition size of $M=31, \epsilon=0$, and a CUDA block dimension of 256. For large $N$, RPTS is approximately 5 times faster than the numerically stable tridiagonal solver of cuSPARSE on the RTX 2080 Ti. For further comparison, the non-pivoting version of cuSPARSE is also shown, which is a hybrid solver of CR and PCR. The absolute performance difference to cuSPARSE diminishes with decreasing $N$, which is partially caused by the decreasing memory throughput for small problem sizes. Although, this is a natural property of GPU memory, which can be seen by the copy kernel performance in Figure 3 , the throughput measurements without any calculation denote the upper performance bound, and indicate that algorithms with higher computational parallelism can further improve the equation throughput, for small problem sizes.

\section{PRECONDITIONING}

Iterative methods to solve sparse linear equation systems have been studied extensively. Nowadays, GPUs are often used in this role. An overview of iterative methods for sparse systems is given for example by Saad [29].

In this section, we compare the ILU(0), Jacobi, and RPTS preconditioners on the GeForce RTX 2080 Ti with each other, and use the GMRES (restart $=20$ ) and BiCGSTAB Krylov solver. Except for RPTS, we use the implementations provided by the MAGMA [3] library and deploy the Incomplete Sparse Approximate Inverse (ISAI) scheme by Anzt et al. [2] with one relaxation step to solve the $L$ and $U$ factors in ILU(0).

We consider the overall matrix weight, the sum of the absolute values of the matrix coefficients $\|A\|_{1,1}:=\sum_{i, j}\left|A_{i, j}\right|$, and define the diagonal weight coverage as

$$
c_{d}(A):=\sum_{i}\left|A_{i, i}\right| /\|A\|_{1,1}
$$




\begin{tabular}{lllrrrrr}
\hline Name & Problem & Origin & DOFs & nnz & mean degree & $c_{d}$ & $c_{t}$ \\
\hline ATMOSMODJ & Fluid Dynamics & SMC & 1270432 & 8814880 & 5.94 & 0.50 & 0.73 \\
ATMOSMODD & Fluid Dynamics & SMC & 1270432 & 8814880 & 5.94 & 0.50 & 0.73 \\
ATMOSMODL & Fluid Dynamics & SMC & 1489752 & 10319760 & 5.93 & 0.50 & 0.63 \\
ECOLOGY1 & 2D/3D & SMC & 1000000 & 4996000 & 4.00 & 0.50 & 0.75 \\
ECOLOGY2 & 2D/3D & SMC & 999999 & 4995991 & 4.00 & 0.50 & 0.75 \\
TRANSPORT & Structural & SMC & 1602111 & 23487281 & 13.67 & 0.50 & 0.75 \\
ANISO1 & 9pt 2D stencil & A & 6250000 & 56220004 & 8.00 & 0.50 & 0.83 \\
ANISO2 & 9pt 2D stencil & A & 6250000 & 56220004 & 8.00 & 0.50 & 0.57 \\
ANISO3 & 9pt 2D stencil & A & 6250000 & 56220004 & 8.00 & 0.50 & 0.83 \\
PFLOw_742 & 2D/3D & SMC & 742793 & 37138461 & 49.00 & 0.16 & 0.24 \\
\hline
\end{tabular}

Table 3: Matrices which are either from the Sparse Matrix Collection [9] (SMC) or are self-created (A). $c_{d}$ and $c_{t}$ represent the diagonal and the tridiagonal weight coverage of the matrix.

and the tridiagonal weight coverage as

$$
c_{t}(A):=\sum_{i}\left(\left|A_{i, i}\right|+\left|A_{i, i-1}\right|+\left|A_{i, i+1}\right|\right) /\|A\|_{1,1},
$$

assuming $A_{N-1, N}=A_{0,-1}=0$.

We want to point out the benefits of the tridiagonal solver as a preconditioner in comparison to the Jacobi preconditioner for anisotropic problems, when the tridiagonal weight coverage is significantly higher than the diagonal weight coverage of the matrix. We use test matrices from the Sparse Matrix Collection [9] and selfconstructed 2D anisotropic problems (see Table 3) on an equidistant grid with the following stencils for matrices ANISO1 and ANISO2:

ANISO1 $=\left(\begin{array}{ccc}-0.2 & -0.1 & -0.2 \\ -1.0 & 3.0 & -1.0 \\ -0.2 & -0.1 & -0.2\end{array}\right)$, ANISO2 $=\left(\begin{array}{ccc}-0.1 & -0.2 & -1.0 \\ -0.2 & 3.0 & -0.2 \\ -1.0 & -0.2 & -0.1\end{array}\right)$.

Matrix ANISO3 is a permutation of matrix ANISO2, such that the strong bands become the first super- and subdiagonal in the matrix and thus are part of the tridiagonal system.

The solution vector $x$ is generated by a sinus function with $x[i]:=\sin (2 \pi f i / N)$, where $N$ is equal to the degrees of freedom of the problem and the frequency is set to $f:=8$. The right-hand side is calculated with the generated solution, and the initial guess for the solution is set to zero.

Figure 5 shows the numerical convergence in terms of iterations. Note that the forward relative error is shown, not the residual, so it is possible that it does not decrease monotonically even for GMRES The results for ILU are missing for matrix PFLOw_742, because the ILU-ISAI implementation of MAGMA is restricted by the amount of non-zero values per matrix row. Not surprisingly, a diagonal preconditioner is weaker than a tridiagonal preconditioner, which is weaker than an ILU preconditioner. However, the ILU preconditioner also requires the longest initialization and execution times. The benefits of the tridiagonal preconditioner in comparison to the Jacobi are visible, if the tridiagonal part of the matrix contains the weights of the anisotropy of the matrix (matrices ANISO1, ANISO3). In contrast, for matrix ANISO2, with $c_{d}=0.5, c_{t}=0.57$, the tridiagonal and Jacobi preconditioner perform equally well.

In Figure 6, the convergence results in terms of execution time are shown. The matrices from the Sparse Matrix Collection are not large enough for RPTS on the large RTX 2080 Ti to run at full speed, e.g. if we compare the achieved equation throughput in Figure 4

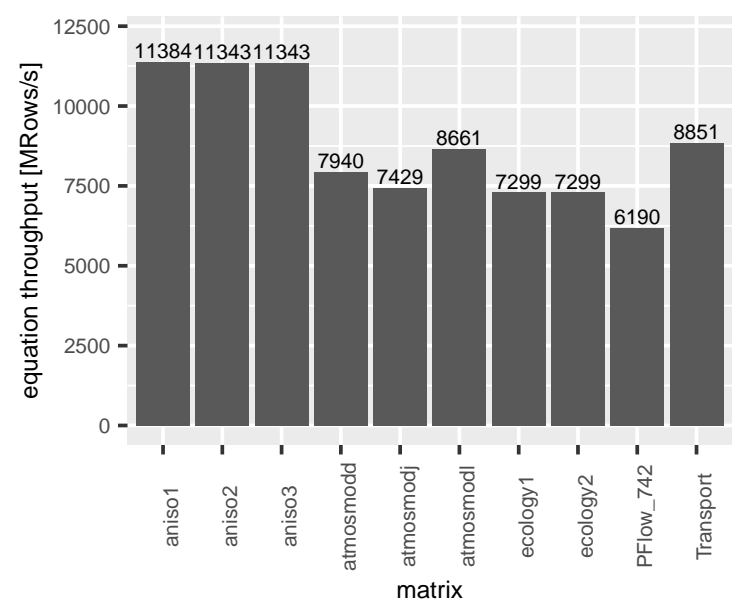

Figure 4: Equation throughput of RPTS in single-precision

with Figure 3, matrix ATMOSMODL is processed with $72 \%$ of the maximum equation throughput on the RTX $2080 \mathrm{Ti}$. Overall we observe that ILU performs worse with BiCGSTAB in comparison to the other preconditioners, because its slow execution consumes a large fraction of the overall time of one BiCGSTAB iteration as shown in Figure 7. When the outer solver is more time-consuming, GMRES contains orthogonalizations, the relative time of the preconditioner decreases and the combination GMRES+ILU benefits from the stronger preconditioning properties of ILU. In the other direction fast preconditioners run better in combination with less complex outer solvers. A second effect is observed for matrices with many non-zero values which cause longer execution times of the sparse matrix-vector product, thus reducing the relative time of the tridiagonal solver as a preconditioner. Hence for matrix PFLOw_742 with BiCGSTAB only $13 \%$ of the time per iteration is spent in RPTS, whereas it is $28 \%$ for the $2 \mathrm{D}$ anisotropic matrices. Even with the low tridiagonal coverage the tridiagonal solver converges faster than Jacobi per iteration on matrix PFLOw_742, however, with the above effect, Jacobi runs faster on time with the Krylov solvers. On the other hand the anisotropic problems execute very quickly with 
preconditioner $\longrightarrow \mathrm{ILU}(0)-\mathrm{ISAl}(1)-$ Jacobi $\longrightarrow$ RPTS

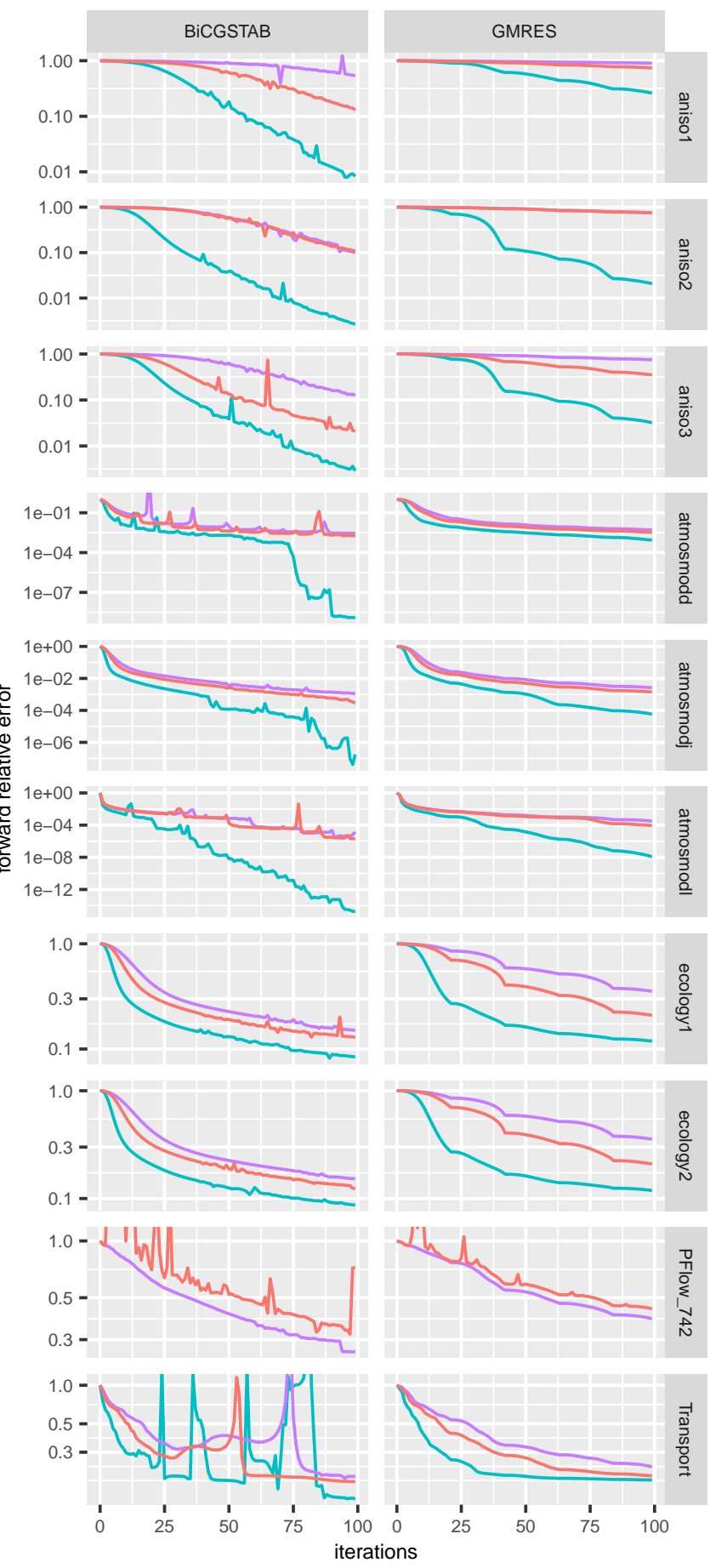

Figure 5: Convergence in double-precision. the tridiagonal preconditioner because $83 \%$ of the matrix weight originates from the tridiagonal part of the matrix.

\section{CONCLUSION}

We have created a hierarchical tridiagonal solver with scaled partial pivoting which fully saturates the memory bandwidth on GPUs. In the CUDA implementation, we employ the technique of on-the-fly data transposition in shared memory, so that adjacent equations can be be processed sequentially by CUDA threads. Although only one or two warps per CUDA block are active during the calculation, the computing time can be entirely hidden behind the data movement because the specific algorithm completely avoids SIMD divergence despite the data dependent pivoting decisions. In comparison to well-established libraries RPTS reaches the same numerical accuracy with a performance which is up to 5 times faster than gtsv2 from cuSPARSE on large problems. We further applied the tridiagonal solver as a preconditioner to Krylov solvers and showed its useability for solving real-world problems in terms of numeric accuracy and performance.

The proposed tridiagonal solver reaches the maximum speed on GPUs. It is so fast that inside Krylov solvers the relative time per iteration spent in tridiagonal preconditioning becomes very small. For the future, this motivates us to develop stronger preconditioners based on tridiagonal solvers.

\section{REFERENCES}

[1] Pablo Alfaro, Pablo Igounet, and Pablo Ezzatti. 2011. A Study on the Implementation of Tridiagonal Systems Solvers Using a GPU. In 2011 30th International Conference of the Chilean Computer Science Society. IEEE, 219-227. https://doi.org/10.1109/SCCC.2011.29

[2] Hartwig Anzt, Thomas K. Huckle, Jürgen Bräckle, and Jack Dongarra. 2018. Incomplete Sparse Approximate Inverses for Parallel Preconditioning. Parallel Comput. 71 (2018), 1-22. https://doi.org/10.1016/j.parco.2017.10.003

[3] Hartwig Anzt, William Sawyer, Stanimire Tomov, Piotr Luszczek, Ichitaro Yamazaki, and Jack Dongarra. 2014. Optimizing Krylov Subspace Solvers on Graphics Processing Units. In Fourth International Workshop on Accelerators and Hybrid Exascale Systems (AsHES), IPDPS 2014. IEEE, IEEE, Phoenix, AZ.

[4] Daniele Bertaccini and Fabio Durastante. 2018. Iterative Methods and Preconditioning for Large and Sparse Linear Systems with Applications. Vol. 369. arXiv:arXiv: $1011.1669 \mathrm{v} 3$

[5] Li-Wen Chang. 2014. Scalable parallel tridiagonal algorithms with diagonal pivoting and their optimization for many-core architectures. Ph.D. Dissertation.

[6] Li Wen Chang, Men Tzung Lo, Nasser Anssari, Ke Hsin Hsu, Norden E. Huang, and Wen Mei W. Hwu. 2011. Parallel implementation of multi-dimensional ensemble empirical mode decomposition. ICASSP, IEEE International Conference on Acoustics, Speech and Signal Processing - Proceedings (2011), 1621-1624. https: //doi.org/10.1109/ICASSP.2011.5946808

[7] Li Wen Chang, John A. Stratton, Hee Seok Kim, and Wen Mei W. Hwu. 2012. A scalable, numerically stable, high-performance tridiagonal solver using GPUs. International Conference for High Performance Computing, Networking, Storage and Analysis, SC (2012). https://doi.org/10.1109/SC.2012.12

[8] Andrew Davidson, Yao Zhang, and John D. Owens. 2011. An auto-tuned method for solving large tridiagonal systems on the GPU. Proceedings - 25th IEEE International Parallel and Distributed Processing Symposium, IPDPS 2011 (2011), 956-965. https://doi.org/10.1109/IPDPS.2011.92

[9] Timothy A. Davis and Yifan Hu. 2011. The University of Florida Sparse Matrix Collection. ACM Trans. Math. Software 38, 1 (2011). https://doi.org/10.1145/ 2049662.2049663

[10] Adrian Perez Dieguez, Margarita Amor, and Ramon Doallo. 2016. New Tridiagonal Systems Solvers on GPU Architectures. Proceedings - 22nd IEEE International Conference on High Performance Computing, HiPC 2015 (2016), 85-93. https: //doi.org/10.1109/HiPC.2015.17

[11] Adrián P. Diéguez, Margarita Amor, and Ramón Doallo. 2019. Parallel prefix operations on GPU: tridiagonal system solvers and scan operators. fournal of Supercomputing 75, 3 (2019), 1510-1523. https://doi.org/10.1007/s11227-0182676-z

[12] Adrián P. Diéguez, Margarita Amor, and Ramón Doallo. 2019. Tree partitioning reduction: A new parallel partition method for solving tridiagonal systems. ACM 
preconditioner $\longrightarrow$ ILU(0)-ISAI $(1) \multimap$ Jacobi $\longrightarrow$ RPTS
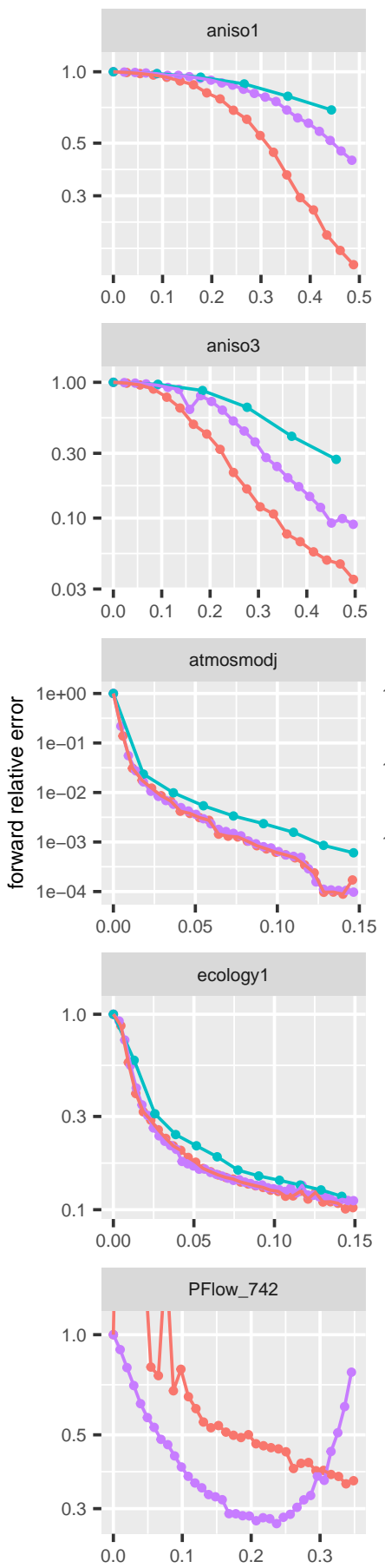
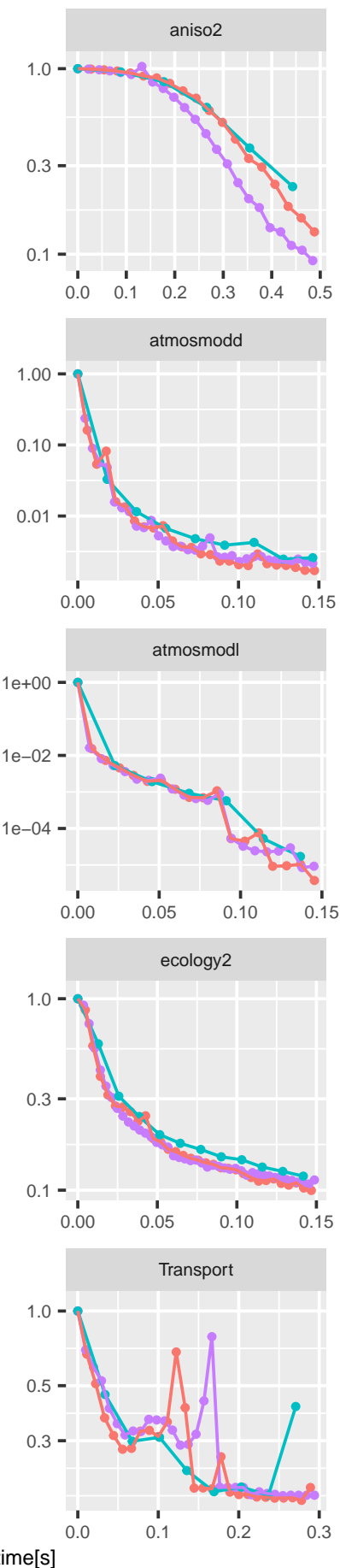

time[s]

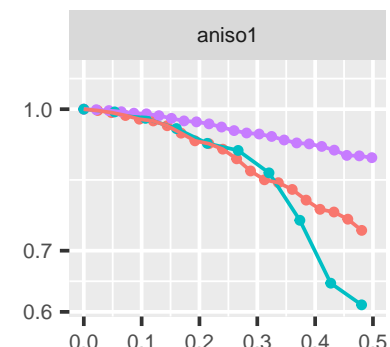

aniso3
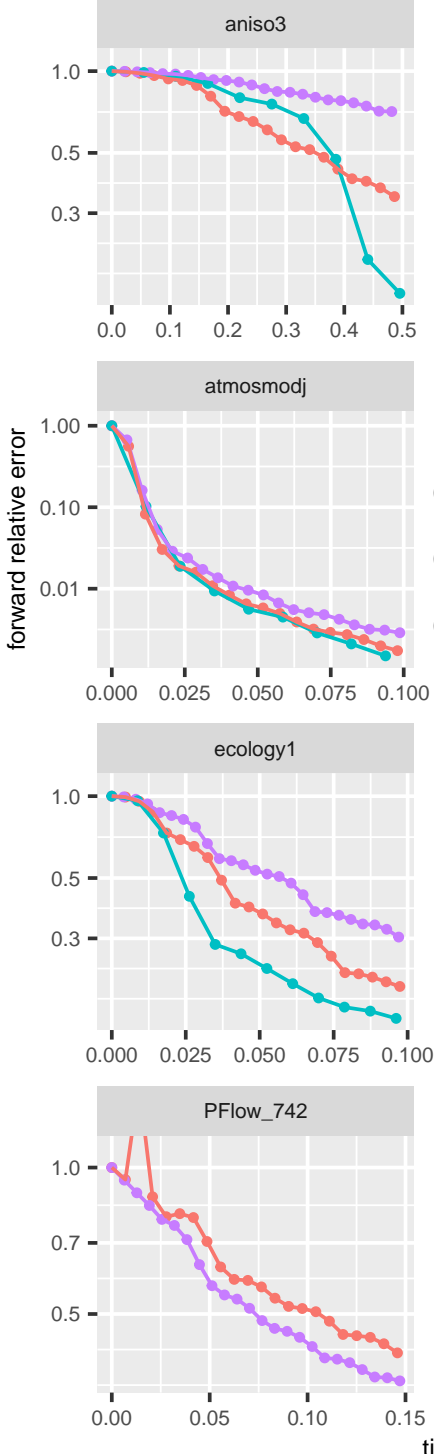

(b) GMRES
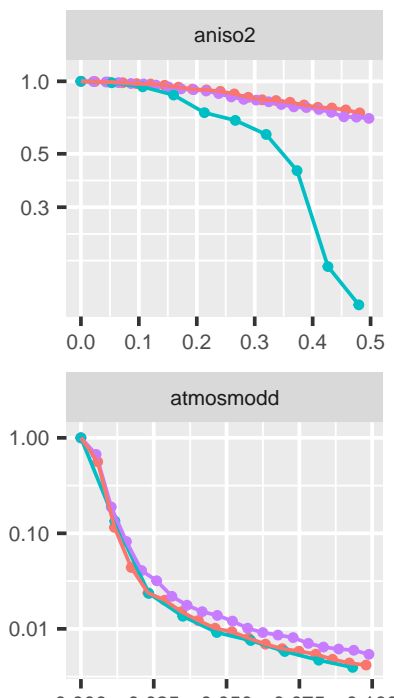

$\begin{array}{ccccc}0.000 & 0.025 & 0.050 & 0.075 & 0.100\end{array}$
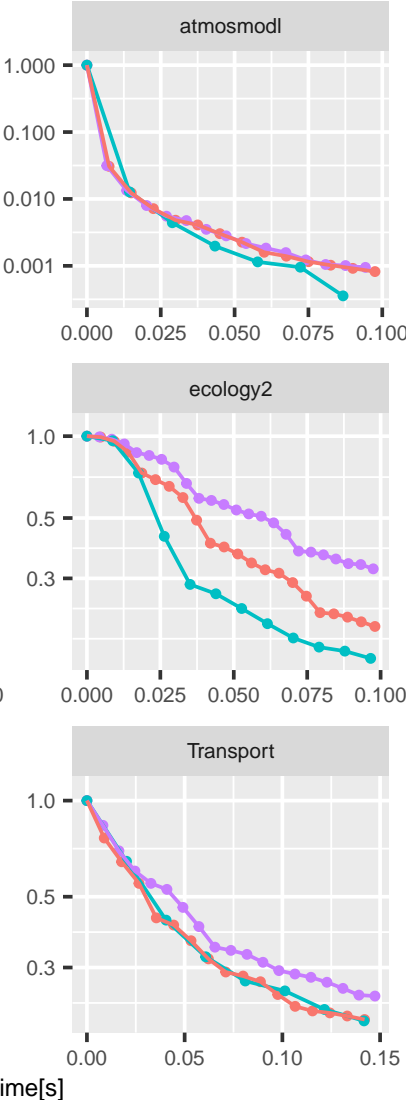

(a) BiCGSTAB

Figure 6: Single-precision convergence performance. A dot represents 5 iterations. 


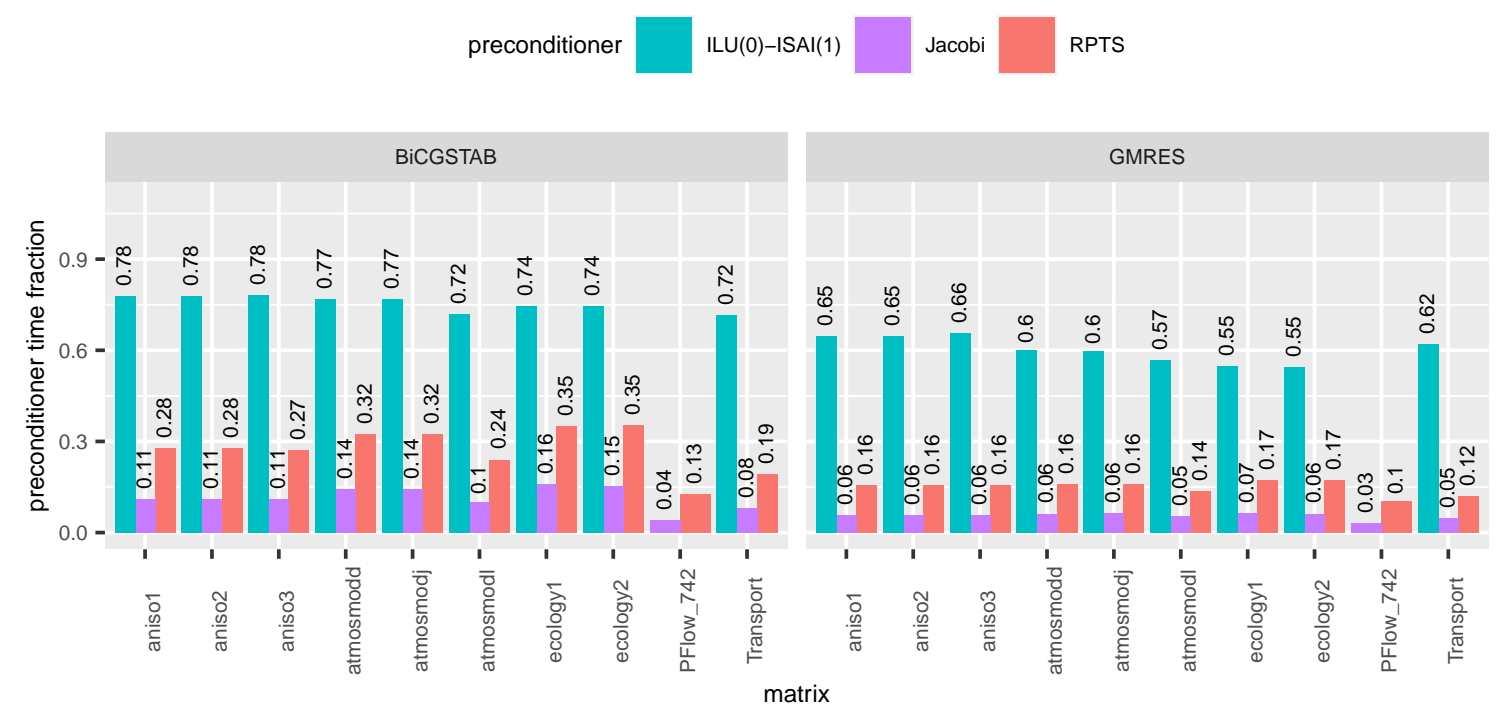

Figure 7: Relative time spent in preconditioner during one solver iteration.

Trans. Math. Software 45, 3 (2019). https://doi.org/10.1145/3328731

[13] Adrián Pérez Diéguez, Margarita Amor, Jacobo Lobeiras, and Ramón Doallo. 2018. Solving large problem sizes of index-digit algorithms on GPU: FFT and tridiagonal system solvers. IEEE Trans. Comput. 67, 1 (2018), 86-101. https //doi.org/10.1109/TC.2017.2723879

[14] Adrian Perez Dieguez, Margarita Amor Lopez, and Ramon Doallo Biempica. 2018. Solving Multiple Tridiagonal Systems on a Multi-GPU Platform. Proceedings 26th Euromicro International Conference on Parallel, Distributed, and NetworkBased Processing, PDP 2018 (2018), 759-763. https://doi.org/10.1109/PDP2018. 2018.00123

[15] Jennifer B. Erway, Roummel F. Marcia, and Joseph A. Tyson. 2010. Generalized diagonal pivoting methods for tridiagonal systems without interchanges. IAENG International fournal of Applied Mathematics 40, 4 (2010), 1-7.

[16] Mike Giles, Endre Laszlo, Istvan Reguly, Jeremy Appleyard, and Julien Demouth. 2014. GPU Implementation of Finite Difference Solvers. Proceedings of WHPCF 2014: 7th Workshop on High Performance Computational Finance Held in conjunction with SC 2014: The International Conference for High Performance Computing, Networking, Storage and Analysis (2014), 1-8. https //doi.org/10.1109/WHPCF.2014.10

[17] Dominik Göddeke and Robert Strzodka. 2011. Cyclic reduction tridiagonal solvers on GPUs applied to mixed-precision multigrid. IEEE Transactions on Parallel and Distributed Systems 23, 1 (2011), 22-32. https://doi.org/10.1109/TPDS.2010.61

[18] Anne Greenbaum. 1997. Iterative methods for solving linear systems.

[19] Gaël Guennebaud, Benoît Jacob, and Others. 2010. Eigen v3. http://eigen.tuxfamily.org.

[20] George R. Halliwell. 2004. Evaluation of vertical coordinate and vertical mixing algorithms in the HYbrid-Coordinate Ocean Model (HYCOM). Ocean Modelling 7, 3-4 (2004), 285-322. https://doi.org/10.1016/j.ocemod.2003.10.002

[21] R.W. Hockney. 1965. A Fast Direct Solution of Poisson's Equation Using Fourier Analysis. 12, 1 (1965), 95-113.

[22] R.W. Hockney and C. R. Jesshope. 1981. Parallel computers : architecture, programming and algorithms. Bristol.

[23] Michael Kass, Aaron Lefohn, and John Owens. 2006. Interactive Depth of Field Using Simulated Diffusion on a GPU. Computing January 2006 (2006), 1-8.

[24] Michael Kass and Gavin Miller. 1990. Rapid, stable fluid dynamics for computer graphics. 24, 4 (1990), 49-57. https://doi.org/10.1145/97879.97884

[25] Hee Seok Kim, Shengzhao Wu, Li Wen Chang, and Wen Mei W. Hwu. 2011. A scalable tridiagonal solver for GPUs. Proceedings of the International Conference on Parallel Processing (2011), 444-453. https://doi.org/10.1109/ICPP.2011.41

[26] Endre László, Mike Giles, and Jeremy Appleyard. 2016. Manycore algorithms for batch scalar and block tridiagonal solvers. ACM Trans. Math. Software 42, 4 (2016). https://doi.org/10.1145/2830568

[27] Jingmei Li, Zhigao Zheng, Qiao Tian, Guoyin Zhang, Fangyuan Zheng, and Yuanyuan Pan. 2017. Research on tridiagonal matrix solver design based on a combination of processors. Computers and Electrical Engineering 62 (2017), 1-16. https://doi.org/10.1016/j.compeleceng.2017.07.014
[28] M. Prieto, R. Santiago, D. Espadas, I. M. Llorente, and F. Tirado. 2001. Parallel Multigrid for Anisotropic Elliptic Equations. 7. Parallel and Distrib. Comput. 61, 1 (2001), 96-114. https://doi.org/10.1006/jpdc.2000.1666

[29] Yousef Saad. 2003. Iterative Methods for Sparse Linear Systems. Iterative Methods for Sparse Linear Systems (2003). https://doi.org/10.1137/1.9780898718003

[30] Milad Souri, Pooria Akbarzadeh, and Hossein Mahmoodi Darian. 2020. Parallel Thomas approach development for solving tridiagonal systems in GPU programming-Steady and unsteady flow simulation. Mechanics and Industry 21, 3 (2020). https://doi.org/10.1051/meca/2020013

[31] Llewellyn Hilleth Thomas. 1949. Elliptic problems in linear difference equations over a network. Watson Sci. Comput. Lab. Rept., Columbia University, New York 1 (1949).

[32] I. E. Venetis, A. Kouris, A. Sobczyk, E. Gallopoulos, and A. H. Sameh. 2015. A direct tridiagonal solver based on Givens rotations for GPU architectures. Parallel Comput. 49 (2015), 101-116. https://doi.org/10.1016/j.parco.2015.03.008

[33] H. H. Wang. 1981. A Parallel Method for Tridiagonal Equations. ACM Transactions on Mathematical Software (TOMS) 7, 2 (1981), 170-183. https://doi.org/10.1145/ 355945.355947

[34] Xiaojing Wang and Z. G. Mou. 1991. A divide-and-conquer method of solving tridiagonal systems on hypercube massively parallel computers. Proceedings of the 3rd IEEE Symposium on Parallel and Distributed Processing 1991 (1991), 810-817. https://doi.org/10.1109/SPDP.1991.218237

[35] Xinliang Wang, Yangtong Xu, and Wei Xue. 2014. A Hierarchical Tridiagonal System Solver for Heterogenous Supercomputers. Proceedings of ScalA 2014: 5th Workshop on Latest Advances in Scalable Algorithms for Large-Scale Systems - held in conjunction with SC 2014: The International Conference for High Performance Computing, Networking, Storage and Analysis (2014), 69-76. https://doi.org/10. 1109/ScalA.2014.12 